\title{
Asymmetric Gaussian Multiple Descriptions and Asymmetric Multilevel Diversity Coding
}

\author{
Soheil Mohajer ${ }^{\dagger}$, Chao Tian ${ }^{\ddagger}$, and Suhas N. Diggavi ${ }^{\dagger}$ \\ $\dagger$ LICOS - EPFL, Lausanne, Switzerland, Email: \{soheil.mohajer, suhas.diggavi\}@epfl.ch \\ $\ddagger$ AT\&T Labs-Research, Florham Park, NJ 07932, USA, Email: tian@research.att.com
}

\begin{abstract}
We consider asymmetric multiple description (MD) source coding for Gaussian source under mean squared error distortion constraints, and focus on the three description problem. Inner and outer bounds for the rate region are derived, both of which can be represented as the intersection of ten half spaces with matching normal directions. Moreover, the gap between the inner and outer bounds is shown to be small.

The inner bound relies on the rate region characterization of a lossless asymmetric multilevel diversity (MLD) coding problem treated in our earlier work, which is a natural generalization of the symmetric MLD coding problem previously considered by Roche et al. Different from symmetric MLD coding, superposition coding is not sufficient in the asymmetric case, and idea akin to network coding needs to be used strategically. Equipped with this finding, and motivated by the connection between symmetric MD and symmetric MLD coding, in this work we consider asymmetric MD as a lossy version of the asymmetric MLD coding, which requires coding beyond simple superposition. An outer bound is also derived, which bears a geometric structure particularly suitable for comparison with the inner bound. Combining the inner and outer bounds provides an approximate characterization of the rate region for the asymmetric Gaussian three description problem.
\end{abstract}

\section{INTRODUCTIONS}

In the multiple description problem (MD) a source is mapped into $K$ descriptions and sent to $2^{K}-1$ decoders, each of which has access to a non-empty subset of the descriptions. There is a distance functions and distortion constraint assigned to each decoder. The decoders are required to reconstruct the source sequence based on their available set of descriptions, such that their approximation be within certain distortion of the original source with respect to their distance function. The goal is to characterize the set of all achievable rates (associated to each description) to satisfy a given set of constraints.

The multiple description problem has been open since its introduction by Wyner et. al. in 1979. At the time El Gamal and Cover [1] deduced an inner bound (EGC bound) for the problem which is restricted to two-description case and single letter distance functions. In a celebrating paper by Ozarow [2], it has been shown that the EGC bound is tight for the Gaussian sources where the distance function is the mean-squared error (MSE) for all the decoders. However, it has been shown by Zhang and Berger [3] that the EGC bound is not tight in general, and the problem is still open except in a few special cases.

In this work we consider the asymmetric three description problem for the Gaussian source under MSE distortion criterion. The result given here is built on two main pillars: the lossless asymmetric multilevel diversity (A-MLD) coding problem [4] and the sum-rate lower bound for symmetric Gaussian MD problem recently discovered in [5].

One important observation leading to this work is the intimate connection between the multilevel diversity (MLD) coding problem and the MD problem. The symmetric MLD (S-MLD) coding problem was considered in [6] and [7]. In [9], we show that for the symmetric MD, achievable rate region based on successive refinement (SR) coding [10] coupled with S-MLD coding, as well as that based on a generalization of the multilayer scheme in [11], provide good approximation to the complete symmetric MD rate region; perhaps more interestingly, these achievable rate regions have the same geometric structure as that of the S-MLD coding rate region. In fact, the S-MLD coding result is essential for establishing the symmetric MD result in [9].

Motivated by this observation, we formulated the lossless A-MLD coding problem in [4], and gave a complete characterization of the three description rate region. The striking difference between S-MLD and A-MLD coding is that superposition coding alone is not sufficient for the asymmetric case, and idea akin to network coding needs to be used. Equipped with this intuition, in this work we first present an achievable region based on SR coding coupled with A-MLD coding. This novel scheme essentially encodes different layers of the SR coded information with linear codes, similarly as in network coding. Such a coding structure has not appeared in the literature for this problem to the best of our knowledge.

For the outer bound, we generalize the sum-rate lower bound for the symmetric $K$ description Gaussian problem in [5]. This bound was derived by generalizing Ozarow's well-known bounding technique in the two-description MD problem [2], and introducing more than one additional auxiliary random variables. Since in the current work we are interested in the rate region, several bounding planes need to be considered, where again the A-MLD coding result provides important guidelines as to the normal directions of these planes, as well as certain conditional independence structure. A similar approach was 


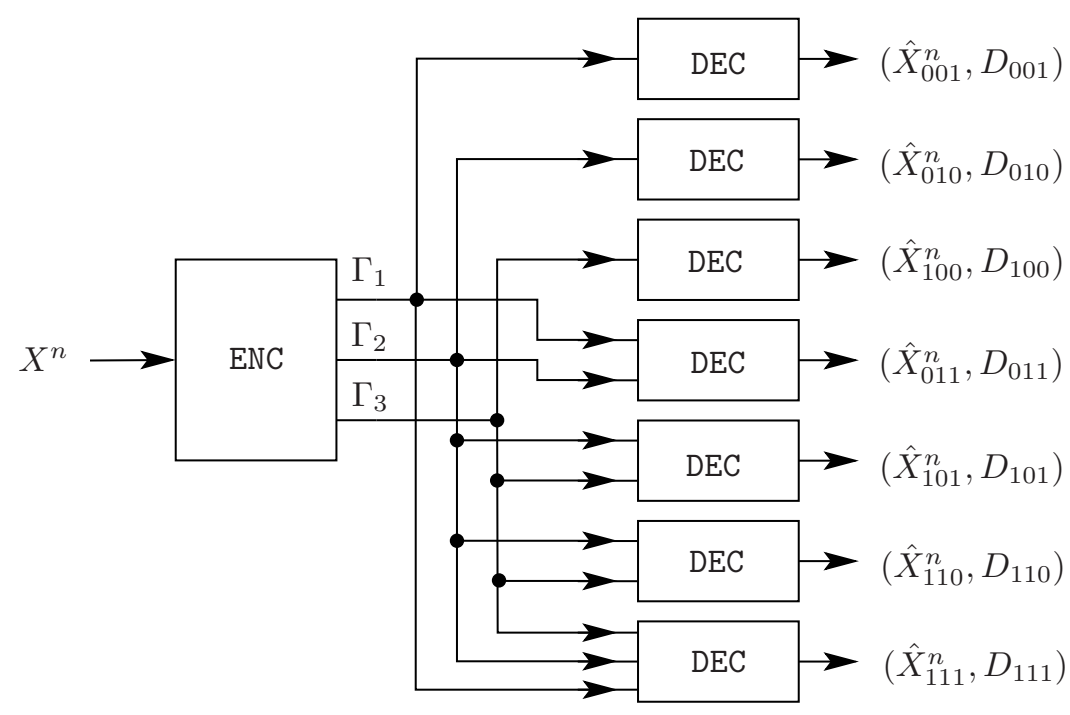

Fig. 1. The three description source coding problem.

used in [9] for the three description symmetric MD, however the asymmetric case requires more effort due to more distortion requirements and the more involved asymmetric geometry.

Both the inner and outer bounds are characterized by ten hyperplanes, and can be compared conveniently. The gap between them is small, and the asymmetric MD rate region is sandwiched in the middle. One surprising consequence of this result is that the proposed simple architecture based on SR and A-MLD coding is in fact not far from optimality. From an engineering viewpoint, this suggests that one can design simple and flexible MD codes that are close to optimality.

\section{NotATIONS AND PROBLEM Formulation}

Let $\left\{X_{k}\right\}$ be a sequence of independent and identically distributed zero mean and unit variance Gaussian random variables with time index $k$. The encoder produces three descriptions of the source, $\Gamma_{1}, \Gamma_{2}$, and $\Gamma_{3}$. There are seven decoders, each of them has access to a non-empty subset of $S=\left\{\Gamma_{1}, \Gamma_{2}, \Gamma_{3}\right\}$ through noiseless bit-pipes. Let $\Omega=\{0,1\}^{3} /\{000\}$, and $\boldsymbol{v} \in \Omega$ be a non-zero binary vector of length three. For simplicity we denote subsets of $S$ by $\mathcal{S}_{\boldsymbol{v}}$, where $\Gamma_{i} \in \mathcal{S}_{\boldsymbol{v}}$ if and only if $\boldsymbol{v}_{i}=1$. Each decoder with descriptions in $\mathcal{S}_{\boldsymbol{v}}$ has to reconstruct the source sequence with a certain level of fidelity with respect to the mean squared error

$$
d\left(x^{n}, \hat{x}_{\boldsymbol{v}}^{n}\right)=\frac{1}{n} \sum_{k=1}^{n}(x(k)-\hat{x} \boldsymbol{v}(k))^{2},
$$

where $\hat{x}_{\boldsymbol{v}}^{n}$ is the reconstructed sequence by the decoder with access to $\mathcal{S}_{\boldsymbol{v}}$. Fig. 1 shows the setting of this problem.

More formally, an $\left(n ; M_{i}, i=1,2,3 ; \Delta \boldsymbol{v}, \boldsymbol{v} \in \Omega\right)$ code is defined as three encoding functions

$$
F_{i}: \mathcal{X}^{n} \longrightarrow\left\{1,2, \ldots, M_{i}\right\}, \quad i=1,2,3,
$$

and seven decoding functions

$$
G \boldsymbol{v}: \prod_{i: \boldsymbol{v}_{i}=1}\left\{1, \ldots, M_{i}\right\} \longrightarrow \mathcal{X}^{n}, \quad \boldsymbol{v} \in \Omega
$$

which satisfy

$$
\mathbb{E} d\left(X^{n}, \hat{X}_{\boldsymbol{v}}^{n}\right)=\Delta \boldsymbol{v}, \quad \forall \boldsymbol{v} \in \Omega
$$

where

$$
\hat{X}_{\boldsymbol{v}}^{n}=G_{\boldsymbol{v}}\left(F_{i}\left(X^{n}\right), i: \boldsymbol{v}_{i}=1\right) .
$$

Here $\prod$ is used to denote set product, and $\mathbb{E}$ is the expectation operator. Throughout this work we assume $\mathcal{X}=\mathbb{R}$.

A triple $\left(R_{1}, R_{2}, R_{3}\right)$ is called $\left(D_{001}, D_{010}, \ldots, D_{111}\right)$-admissible if for every $\varepsilon>0$ and sufficiently large $n$, there exists a code $\left(n ; M_{i}, i=1,2,3 ; \Delta \boldsymbol{v}, \boldsymbol{v} \in \Omega\right)$ such that

$$
\frac{1}{n} \log \left|M_{i}\right| \leq R_{i}+\varepsilon, \quad i=1,2,3,
$$


and

$$
\Delta \boldsymbol{v} \leq D_{\boldsymbol{v}}+\varepsilon, \quad \boldsymbol{v} \in \Omega
$$

Let $\mathcal{R}(\mathbf{D})$ be the set of all $\mathbf{D}$-admissible rate triple, where $\mathbf{D}=\left(D_{001}, D_{010}, \ldots, D_{111}\right)$. In fact, this is the region we seek to characterize.

Our approach to this problem to establish a new outer bound for the admissible region. Comparing our outer bound to a simple known inner bound shows that the gap between the bounds is at most a constant bit per description. This gives us an approximate characterization for the admissible rate region.

Let $\boldsymbol{u}, \boldsymbol{v} \in \Omega$ be two indicator vectors. Define $\boldsymbol{u} \preceq \boldsymbol{v}$ if and only if $\boldsymbol{u}_{i} \leq \boldsymbol{v}_{i}$ for $i=1,2,3$. For a given pair $\boldsymbol{u} \preceq \boldsymbol{v}$, it is clear that the decoder with access to $\mathcal{S}_{\boldsymbol{v}}$ can reconstruct the source sequence with less distortion than the one with access to $\mathcal{S}_{\boldsymbol{u}}$, even if $D \boldsymbol{v} \leq D \boldsymbol{u}$. This suggests to define a modified distortion vector $\tilde{\mathbf{D}}$, which is intuitively the actual distortion constraints which are satisfied without further refinement.

Definition 1. For a given distortion vector $\mathbf{D}$, its modification is the vector $\tilde{\mathbf{D}}=\left(\tilde{D}_{001}, \tilde{D}_{010}, \ldots, \tilde{D}_{111}\right)$, where

$$
\tilde{D}_{\boldsymbol{v}}=\min _{\boldsymbol{u}: \boldsymbol{u} \preceq \boldsymbol{v}} D \boldsymbol{v}
$$

Lemma 1. $\mathcal{R}(\tilde{\mathbf{D}})=\mathcal{R}(\mathbf{D})$.

Proof of Lemma 1: It is clear that $\tilde{D} \boldsymbol{v} \leq D \boldsymbol{v}$ for all $\boldsymbol{v} \in \Omega$, and therefore $\mathcal{R}(\tilde{\mathbf{D}}) \subseteq \mathcal{R}(\mathbf{D})$. So, it remains to prove $\mathcal{R}(\mathbf{D}) \subseteq \mathcal{R}(\tilde{\mathbf{D}})$. Let $\left(R_{1}, R_{2}, R_{3}\right) \in \mathcal{R}(\mathbf{D})$ be an admissible rate triple for $\mathbf{D}$, and $\left(n ; M_{i} ; \Delta_{\boldsymbol{v}}\right)$ be a code which achieves the distortion constraints, with encoding functions $\left\{F_{i}\right\}$, and decoding functions $\{G \boldsymbol{v}\}$. Define

$$
\tilde{x}_{\boldsymbol{v}}^{n}=\tilde{G} \boldsymbol{v}\left(F_{i}\left(x^{n}\right), i: \boldsymbol{v}_{i}=1\right) \triangleq \arg \min _{\boldsymbol{u}: \boldsymbol{u} \preceq \boldsymbol{v}} d\left(x^{n}, \hat{x}_{\boldsymbol{u}}^{n}\right)
$$

Obviously,

$$
\mathbb{E} d\left(X^{n}, \tilde{X}_{\boldsymbol{v}}^{n}\right)=\mathbb{E} \min _{\boldsymbol{u}: \boldsymbol{u} \preceq \boldsymbol{v}} d\left(X^{n}, \tilde{X}_{\boldsymbol{u}}^{n}\right) \leq \min _{\boldsymbol{u}: \boldsymbol{u} \preceq \boldsymbol{v}} \mathbb{E} d\left(X^{n}, \tilde{X}_{\boldsymbol{u}}^{n}\right) \leq \tilde{D} \boldsymbol{v}+\varepsilon .
$$

Thus the similar code with the modified decoding functions satisfy the modified constraints, and $\left(R_{1}, R_{2}, R_{3}\right) \in \mathcal{R}(\tilde{\mathbf{D}})$.

Result of Lemma 1 shows that without loss of generality, we shall assume the distortion constraints are given such that $D_{\boldsymbol{v}} \leq \min \boldsymbol{u}: \boldsymbol{u} \preceq \boldsymbol{v} D \boldsymbol{u}$.

For the purpose of characterizing $\mathcal{R}(\mathbf{D})$, we may assume $1 \geq D_{001} \geq D_{010} \geq D_{\boldsymbol{v}}$ for all $\boldsymbol{v} \neq 001,010$, $i$. e., the two largest distortion constraints are corresponded to two single description decoders with access to $\Gamma_{1}$ and $\Gamma_{2}$, respectively. The concept of ordering can be introduced as follows.

Definition 2. An ordering of the decoders is an one-to-one mapping $\mathscr{L}: \Omega \longrightarrow\{1,2, \ldots, 7\}$, which satisfies

(i) $\mathscr{L}(001)=1$ and $\mathscr{L}(010)=2$,

(ii) $\mathscr{L}(\boldsymbol{v})>\mathscr{L}(\boldsymbol{u})$ implies $D \boldsymbol{v} \leq D \boldsymbol{u}^{1}$.

Sometimes we shall refer to the resulted value of $\mathscr{L}(\cdot)$ as level. There are eight different orderings for the three descriptions problem. In this work we focus on the ordering $\mathscr{L}(100)=3, \mathscr{L}(011)=4, \mathscr{L}(101)=5, \mathscr{L}(110)=6$, and $\mathscr{L}(111)=7$. However, the result can be extended to all other seven possible orderings, which is omitted for brevity.

\section{THE INNER BOUND}

In this section we present an inner bound for $\mathcal{R}(\mathbf{D})$. The achievability scheme is based on a simple strategy: first we produce an SR coded bitstream from the source such that the $k$-th level distortion constraint is satisfied with the first $k$ layers, and then a lossless coding component is used to generate the descriptions. Since the latter component relies on the A-MLD coding result in [4], we first give a brief review.

\section{A. A brief review of A-MLD coding}

Let $\mathbf{V}_{1}^{7}=\left\{\left(V_{1}^{n}, V_{2}^{n}, V_{3}^{n}, V_{4}^{n}, V_{5}^{n}, V_{6}^{n}, V_{7}^{n}\right)\right\}$ be seven independent source sequences. An encoder encodes the source sequences into three descriptions $\Gamma_{i}, i=1,2,3$, at respective rates $R_{i}, i=1,2,3$, such that a decoder with access to the descriptions $S \boldsymbol{v}$ can losslessly decode $\left(V_{1}^{n}, \ldots, V_{\mathscr{L}(\boldsymbol{v})}\right)$, for $\boldsymbol{v} \in \Omega$. The problem is to characterize achievable rate triples $\left(R_{1}, R_{2}, R_{3}\right)$ in terms of $h_{i}=H\left(V_{i}\right)$. The following theorem is the main result in [4].

\footnotetext{
${ }^{1}$ We may use both $D \boldsymbol{v}$ and $D_{\mathscr{L}(\boldsymbol{v})}$ to denote the distortion constraint at the decoder $\boldsymbol{v}$.
} 


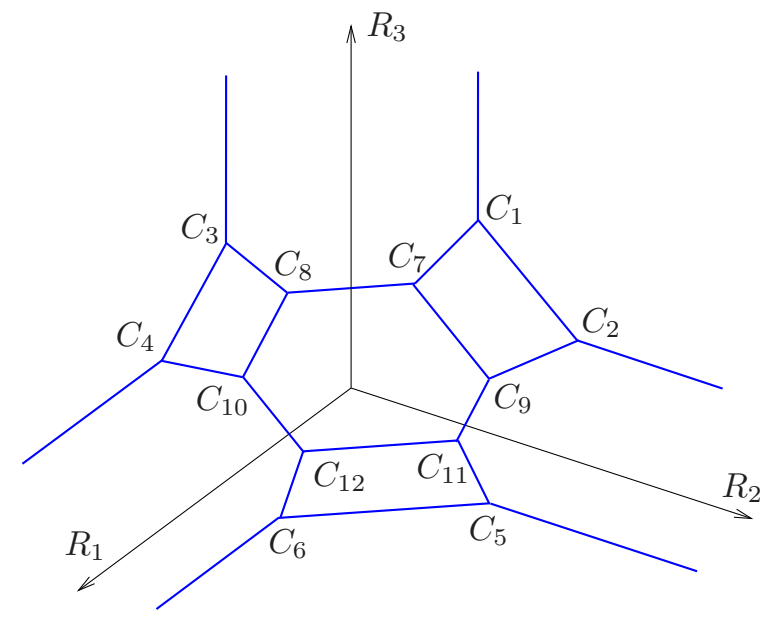

Fig. 2. Admissible rate region of the lossless problem for the case $h_{4} \leq h_{3} \leq h_{4}+h_{5}$

Theorem 1 ( [4] Theorem 1). A rate triple $\left(R_{1}, R_{2}, R_{3}\right)$ is achievable for A-MLD coding if and only if

$$
\begin{aligned}
R_{1} & \geq H_{1} \\
R_{2} & \geq H_{2} \\
R_{3} & \geq H_{3} \\
R_{1}+R_{2} & \geq H_{1}+H_{4} \\
R_{2}+R_{3} & \geq H_{1}+H_{5} \\
R_{2}+R_{3} & \geq H_{2}+H_{6} \\
2 R_{1}+R_{2}+R_{3} & \geq 2 H_{1}+H_{4}+H_{7} \\
R_{1}+2 R_{2}+R_{3} & \geq H_{1}+H_{2}+H_{4}+H_{7} \\
R_{1}+R_{2}+2 R_{3} & \geq H_{1}+H_{2}+H_{3}+H_{7}+\max \left\{h_{3}, h_{4}+h_{5}\right\} \\
R_{1}+R_{2}+R_{3} & \geq H_{1}+H_{2}+\frac{1}{2} h_{3}+H_{7}+\frac{1}{2} \max \left\{h_{3}, h_{4}\right\},
\end{aligned}
$$

where $H_{i} \triangleq \sum_{j=1}^{i} h_{j}$.

According to the proportion of $h_{3}, h_{4}$, and $h_{5}$, we can characterize the problem in three different regimes. Fig. 2 shows the rate region for the regime $h_{4} \leq h_{3} \leq h_{4}+h_{5}$ under the same ordering considered in this work.

It is worth considering one particular corner point, e.g. $C_{12}$, which corresponds to the rate triple

$$
\left(R_{1}, R_{2}, R_{3}\right)=\left(H_{1}+h_{3}+h_{7}, H_{3}+h_{6}, H_{2}+h_{4}+h_{5}\right) .
$$

To achieve this point we compress each source losslessly into a binary representation $\bar{V}_{i}$ of length $\ell_{i}$ which is approximately $n h_{i}$, respectively, then perform the follow operations. First partition $\bar{V}_{3}$ into $\bar{V}_{3,1}$ and $\bar{V}_{3,2}$, of lengths $\ell_{3}-\ell_{4}$ and $\ell_{4}$, respectively. Next $\bar{V}_{5}$ is partitioned into $\bar{V}_{5,1}$ and $\bar{V}_{5,2}$ of lengths $\ell_{3}-\ell_{4}$ and $\ell_{4}+\ell_{5}-\ell_{3}$, respectively. The three descriptions are then given by

$$
\begin{aligned}
& \Gamma_{1}: \bar{V}_{1}, \bar{V}_{4}, \bar{V}_{5,1}, \bar{V}_{7} \\
& \Gamma_{2}: \bar{V}_{1}, \bar{V}_{2}, \bar{V}_{3,1} \oplus \bar{V}_{5,1}, \bar{V}_{3,2} \oplus \bar{V}_{4}, \bar{V}_{6} \\
& \Gamma_{3}: \bar{V}_{1}, \bar{V}_{2}, \bar{V}_{3}, \bar{V}_{5,2},
\end{aligned}
$$

where $\oplus$ is the binary XOR operation. By the coding requirement, $\Gamma_{1}$ and $\Gamma_{2}$ can jointly reconstruct $V_{1}, V_{2}, V_{3}, V_{4}$. Notice that $\bar{V}_{3}$ was never explicitly given in $\Gamma_{1}$ or $\Gamma_{2}$, yet by proper linear operation, it can be readily recovered. Fig. 3 illustrates this linear encoding scheme. 


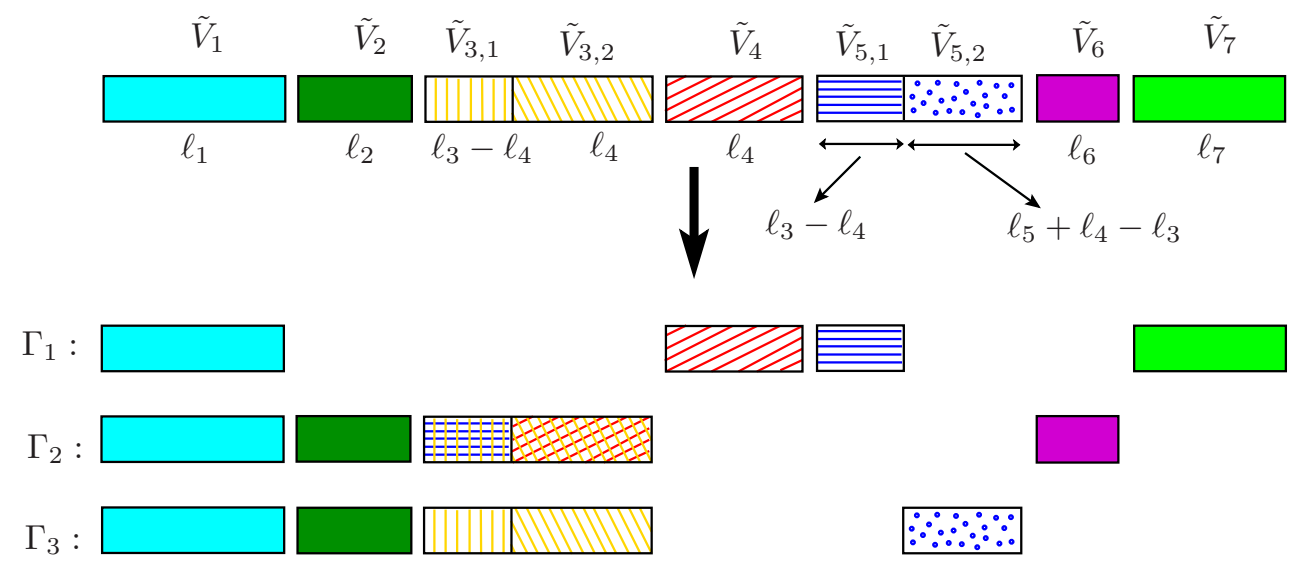

Fig. 3. Linear encoding for the corner-point $C_{12}$

\section{B. An achievable rate region}

For a given distortion vector $\mathbf{D}=\left(D_{1} \ldots, D_{7}\right)=\left(D_{\mathscr{L}^{-1}(1)}, D_{\mathscr{L}^{-1}(2)}, \ldots, D_{\mathscr{L}^{-1}(7)}\right)$, let $\overline{\mathcal{R}}(\mathbf{D})$ be the set of rate triples $\left(R_{1}, R_{2}, R_{3}\right)$ satisfying

$$
\begin{aligned}
R_{1} & \geq \frac{1}{2} \log \frac{1}{D_{1}} \\
R_{2} & \geq \frac{1}{2} \log \frac{1}{D_{2}} \\
R_{3} & \geq \frac{1}{2} \log \frac{1}{D_{3}} \\
R_{1}+R_{2} & \geq \frac{1}{2} \log \frac{1}{D_{1} D_{4}} \\
R_{1}+R_{3} & \geq \frac{1}{2} \log \frac{1}{D_{1} D_{5}} \\
R_{2}+R_{3} & \geq \frac{1}{2} \log \frac{1}{D_{2} D_{6}} \\
2 R_{1}+R_{2}+R_{3} & \geq \frac{1}{2} \log \frac{1}{D_{1}^{2} D_{4} D_{7}} \\
R_{1}+2 R_{2}+R_{3} & \geq \frac{1}{2} \log \frac{1}{D_{1} D_{2} D_{4} D_{7}} \\
R_{1}+R_{2}+2 R_{3} & \geq \max \left\{\frac{1}{2} \log \frac{1}{D_{1} D_{2} D_{5} D_{7}}, \frac{1}{2} \log \frac{1}{D_{1} D_{3}^{2} D_{7}}\right\} \\
R_{1}+R_{2}+R_{3} & \geq \max \left\{\frac{1}{2} \log \frac{1}{D_{1} D_{3} D_{7}}, \frac{1}{4} \log \frac{1}{D_{1}^{2} D_{2} D_{4} D_{7}^{2}}\right\} .
\end{aligned}
$$

The following theorem gives a simple inner bound for the admissible rate region.

\section{Theorem 2.}

$$
\overline{\mathcal{R}}(\mathbf{D}) \subseteq \mathcal{R}(\mathbf{D})
$$

Proof of Lemma 2: The proof of this theorem is straightforward and based on the successive refinability of Gaussian sources [10]. In the encoding scheme, we first produce seven successive refinement layers of the source, ad then encode them losslessly. Let $\Psi_{k}$ be the $k$-th SR layer, such that having $\Psi_{1}, \ldots, \Psi_{k}$, the decoder is able to reconstruct the source within distortion constraint $D_{k}$. Therefore, $\Psi_{k}$ can be encoded to a bitstream of length

$$
n h_{k} \triangleq n R\left(D_{k}\right)-n R\left(D_{k-1}\right)
$$

where $R(D)=-\frac{1}{2} \log D$ is the unit variance Gaussian R-D function, and $D_{0} \triangleq 1$. Now, it is only remained to losslessly encode the obtained (bitstream) SR layers using into three descriptions. Theorem 1 characterizes the achievable rate triples of such encoding. Substituting $h_{i}, i=1, \ldots, 7$, from (2) into (P1)-(P10) gives the result. 
For the case $D_{2} D_{5} \leq D_{3}^{2} \leq D_{2} D_{4}$, consider the rate triple $\left(R_{1}, R_{2}, R_{3}\right)=\left(\frac{1}{2} \log \frac{D_{2} D_{6}}{D_{1} D_{3} D_{7}}, \frac{1}{2} \log \frac{D_{5}}{D_{3} D_{6}}, \frac{1}{2} \log \frac{D_{3}}{D_{2} D_{5}}\right)$, which corresponds to $C_{12}$ in the A-MLD coding case. Clearly, the coding scheme for this point matches that for $C_{12}$ closely, and the SR encoded information in the 3-rd, 4-th and 5-th layers needs to be strategically re-processed using linear codes. Without the underlining A-MLD coding scheme, it is difficult, if not impossible, to devise this coding operation directly.

\section{The Outer Bound}

In this section we provide an outer bound for the set of admissible rates $\mathcal{R}(\mathbf{D})$.

For a given distortion vector $\mathbf{D}=\left(D_{1} \ldots, D_{7}\right)=\left(D_{\mathscr{L}^{-1}(1)}, D_{\mathscr{L}^{-1}(2)}, \ldots, D_{\mathscr{L}^{-1}(7)}\right)$, let $\underline{\mathcal{R}}(\mathbf{D})$ be the set of rate triples $\left(R_{1}, R_{2}, R_{3}\right)$ satisfying

$$
\begin{aligned}
R_{1} & \geq \frac{1}{2} \log \frac{1}{D_{1}} \\
R_{2} & \geq \frac{1}{2} \log \frac{1}{D_{2}} \\
R_{3} & \geq \frac{1}{2} \log \frac{1}{D_{3}} \\
R_{1}+R_{2} & \geq-1+\frac{1}{2} \log \frac{1}{D_{1} D_{4}} \\
R_{1}+R_{3} & \geq-1+\frac{1}{2} \log \frac{1}{D_{1} D_{5}} \\
R_{2}+R_{3} & \geq-1+\frac{1}{2} \log \frac{1}{D_{2} D_{6}} \\
2 R_{1}+R_{2}+R_{3} & \geq-3+\frac{1}{2} \log \frac{1}{D_{1}^{2} D_{4} D_{7}} \\
R_{1}+2 R_{2}+R_{3} & \geq-3+\frac{1}{2} \log \frac{1}{D_{1} D_{2} D_{4} D_{7}} \\
R_{1}+R_{2}+2 R_{3} & \geq \max \left\{-3+\frac{1}{2} \log \frac{1}{D_{1} D_{2} D_{5} D_{7}},-\frac{5}{2}+\frac{1}{2} \log \frac{1}{D_{1} D_{3}^{2} D_{7}}\right\} \\
R_{1}+R_{2}+R_{3} & \geq \max \left\{-2+\frac{1}{2} \log \frac{1}{D_{1} D_{3} D_{7}},-\frac{1}{4}+\frac{1}{4} \log \frac{1}{D_{1}^{2} D_{2} D_{4} D_{7}^{2}}\right\} .
\end{aligned}
$$

The following theorem establishes the outer bound on the admissible rate tuples.

\section{Theorem 3.}

$$
\mathcal{R}(\mathbf{D}) \subseteq \underline{\mathcal{R}}(\mathbf{D})
$$

As we stated before, the proof of this theorem is based on the idea of multilevel diversity coding. We will need to define a set of auxiliary random variables in order to prove the theorem, which are some noisy versions of the source. Let $N_{i} \sim$ $\mathcal{N}\left(0, \sigma_{i}^{2}\right), i=1, \ldots, 6$, be mutually independent zero-mean Gaussian random variables with variance $\sigma_{i}^{2}$. They are also assumed to be independent of $X$. Such a strategy with a single auxiliary variable was used to characterize the two descriptions Gaussian MD region [2]. A noisy version of the source, $Y_{i}$, is defined as

$$
Y_{i}=X+Z_{i}, \quad i=1, \ldots, 6
$$

where $Z_{i}=\sum_{j=i}^{6} N_{j}$ for $j=1, \ldots, 6$. Thus $d_{i} \triangleq \sum_{j=i}^{6} \sigma_{j}^{2}$ would be the variance of the noises $Z_{i}$, for $i=1, \ldots, 6$. We also define $d_{7}=0$ and $Y_{7}=X$ for convenience. Note that these variables are corrupted by incremental noises and therefore, form a Markov chain as $\left(\Gamma_{1}, \Gamma_{2}, \Gamma_{3}\right) \leftrightarrow X^{n} \leftrightarrow Y_{6}^{n} \leftrightarrow Y_{5}^{n} \leftrightarrow \ldots \leftrightarrow Y_{1}^{n}$

The following two lemmas are extracted from the result in [5], and are rewritten here for convenient application in the proof for the outer bound.

Lemma 2. For any set of descriptions $\mathcal{S}_{\boldsymbol{v}}$, and noisy version of the source $Y_{i}$, we have

$$
I\left(\mathcal{S}_{\boldsymbol{v}} ; Y_{i}^{n}\right) \geq \frac{n}{2} \log \frac{1+d_{i}}{D_{\boldsymbol{v}}+d_{i}}
$$


Proof of Lemma 2:

$$
\begin{aligned}
I\left(\mathcal{S}_{\boldsymbol{v}} ; Y_{i}^{n}\right) & =h\left(Y_{i}^{n}\right)-h\left(Y_{i}^{n} \mid \mathcal{S}_{\boldsymbol{v}}\right) \\
& =h\left(Y_{i}^{n}\right)-h\left(Y_{i}^{n}-\hat{X}_{\boldsymbol{v}}^{n} \mid \mathcal{S}_{\boldsymbol{v}}\right) \\
& \geq h\left(Y_{i}^{n}\right)-h\left(X^{n}+Z_{i}^{n}-\hat{X}_{\boldsymbol{v}}^{n}\right) \\
& \geq h\left(Y_{i}^{n}\right)-\sum_{t=1}^{n} h\left(X(t)-\hat{X}_{\boldsymbol{v}}(t)+Z_{j}(t)\right) \\
& (a) \frac{n}{2} \log \left(2 \pi e\left(1+d_{i}\right)\right)-\sum_{t=1}^{n} \frac{1}{2} \log \left(2 \pi e\left(\mathbb{E}\left(X(t)-\hat{X}_{\boldsymbol{v}}(t)\right)^{2}+d_{i}\right)\right) \\
& \left(\text { b) } \frac{n}{2} \log \left(1+d_{i}\right)-\frac{n}{2} \log \left(\mathbb{E} d\left(X^{n}, \hat{X}_{\boldsymbol{v}}^{n}\right)+d_{i}\right)\right. \\
& \left(\text { c) } \frac{n}{2} \log \frac{1+d_{i}}{D \boldsymbol{v}+d_{i}}\right.
\end{aligned}
$$

where $(a)$ is due to the fact that the entropy of any random variable is upper bounded by that of Gaussian variables with the same variance; $(b)$ is implied by concavity of the function $\log (x)$; and in $(c)$ we have used the fact that $\log (x+a)$ is an increasing function in $x$.

Lemma 3. For any subset of the descriptions $\mathcal{S}_{\boldsymbol{v}}$, and two noisy versions of the source $Y_{i}$ and $Y_{j}$ with $i<j$, we have

$$
I\left(\mathcal{S}_{\boldsymbol{v}} ; Y_{j}^{n}\right)-I\left(\mathcal{S}_{\boldsymbol{v}} ; Y_{i}^{n}\right) \geq \frac{n}{2} \log \frac{\left(1+d_{j}\right)\left(D \boldsymbol{v}+d_{i}\right)}{\left(1+d_{i}\right)\left(D \boldsymbol{v}+d_{j}\right)} .
$$

Proof of Lemma 3: Note that

$$
\begin{aligned}
h\left(Y_{i}^{n} \mid \mathcal{S}_{\boldsymbol{v}}\right)-h\left(Y_{j}^{n} \mid \mathcal{S}_{\boldsymbol{v}}\right) & \stackrel{(a)}{=} h\left(Y_{i}^{n} \mid \mathcal{S}_{\boldsymbol{v}}\right)-h\left(Y_{j}^{n} \mid \mathcal{S}_{\boldsymbol{v}}, Z_{i}^{n}-Z_{j}^{n}\right) \\
& \stackrel{(b)}{=} h\left(Y_{i}^{n} \mid \mathcal{S}_{\boldsymbol{v}}\right)-h\left(Y_{i}^{n} \mid \mathcal{S}_{\boldsymbol{v}}, Z_{i}^{n}-Z_{j}^{n}\right) \\
& =I\left(Y_{i}^{n} ; Z_{i}^{n}-Z_{j}^{n} \mid \mathcal{S}_{\boldsymbol{v}}\right) \\
& =h\left(Z_{i}^{n}-Z_{j}^{n} \mid \mathcal{S}_{\boldsymbol{v}}\right)-h\left(Z_{i}^{n}-Z_{j}^{n} \mid \mathcal{S}_{\boldsymbol{v}}, Y_{i}^{n}\right) \\
& \stackrel{(c)}{\geq} h\left(Z_{i}^{n}-Z_{j}^{n}\right)-h\left(Z_{i}^{n}-Z_{j}^{n} \mid Y_{i}^{n}-\hat{X}_{\boldsymbol{v}}^{n}\right) \\
& \geq \sum_{t=1}^{n} h\left(Z_{i}(t)-Z_{j}(t)\right)-h\left(Z_{i}(t)-Z_{j}(t) \mid Y_{i}(t)-\hat{X}_{\boldsymbol{v}}(t)\right) \\
& =\sum_{t=1}^{n} I\left(Z_{i}(t)-Z_{j}(t) ; X(t)-\hat{X}_{\boldsymbol{v}}(t)+Z_{i}(t)\right) \\
& (d) \\
& \geq \sum_{t=1}^{n} \frac{1}{2} \log \frac{\mathbb{E}\left(X(t)-\hat{X}_{\boldsymbol{v}}(t)\right)^{2}+d_{i}}{\mathbb{E}\left(X(t)-\hat{X}_{\boldsymbol{v}}(t)\right)^{2}+d_{j}} \\
& \stackrel{(e)}{\geq} \frac{n}{2} \log \frac{D_{\boldsymbol{v}}+d_{i}}{D_{\boldsymbol{v}}+d_{j}}
\end{aligned}
$$

where $(a)$ holds because $Y_{j}^{n}$ is independent of $Z_{i}^{n}-Z_{j}^{n}=N_{i}^{n}+\cdots+N_{j-1}^{n}$; (b) is due to $Y_{i}=Y_{j}+\left(Z_{i}-Z_{j}\right)$; the data processing inequality implies $(c)$; in $(d)$ we use the worst noise lemma in [8], [12]; and $(e)$ is due to convexity and monotonicity of $\log (x+a) /(x+b)$ in $x$ when $a \geq b$. Therefore, we simply have

$$
\begin{aligned}
I\left(\mathcal{S}_{\boldsymbol{v}} ; Y_{j}^{n}\right)-I\left(\mathcal{S}_{\boldsymbol{v}} ; Y_{i}^{n}\right) & =h\left(Y_{j}^{n}\right)-h\left(Y_{j}^{n} \mid \mathcal{S}_{\boldsymbol{v}}\right)-h\left(Y_{i}^{n}\right)+h\left(Y_{i}^{n} \mid \mathcal{S}_{\boldsymbol{v}}\right) \\
& \geq \frac{n}{2} \log \frac{1+d_{j}}{1+d_{i}}+\frac{n}{2} \log \frac{D \boldsymbol{v}+d_{i}}{D_{\boldsymbol{v}}+d_{j}} \\
& =\frac{n}{2} \log \frac{\left(1+d_{j}\right)\left(D \boldsymbol{v}+d_{i}\right)}{\left(1+d_{i}\right)\left(D \boldsymbol{v}+d_{j}\right)}
\end{aligned}
$$

Now we are ready to prove Theorem 3.

Proof of Theorem 3: In this proof, it is sufficient to choose $d_{i}=D_{i}$, for $i=1, \ldots, 6$. The single description levels inequalities are just straight forward result of Lemma 2.

$$
n R_{i} \geq H\left(\mathcal{S}_{\mathscr{L}^{-1}(i)}\right)=H\left(\mathcal{S}_{\mathscr{L}^{-1}(i)}\right)-H\left(\mathcal{S}_{\mathscr{L}^{-1}(i)} \mid X^{n}\right)=I\left(\mathcal{S}_{\mathscr{L}^{-1}(i)} ; X^{n}\right) \geq \frac{n}{2} \log \frac{1}{D_{i}}
$$


where we used Lemma 2 for $Y_{7}=X$ with $d_{7}=0$ in the last inequality. This proves (L1)-(L3).

The bound for the two description rates in (L4) follows from

$$
\begin{aligned}
n\left(R_{1}+R_{2}+2 \varepsilon\right) & \geq H\left(\mathcal{S}_{001}\right)+H\left(\mathcal{S}_{010}\right) \\
& (a) H\left(\mathcal{S}_{001}\right)+H\left(\mathcal{S}_{010}\right)-H\left(\mathcal{S}_{011} \mid X^{n}\right)-\left[H\left(\mathcal{S}_{001} \mid Y_{2}^{n}\right)+H\left(\mathcal{S}_{010} \mid Y_{2}^{n}\right)-H\left(\mathcal{S}_{011} \mid Y_{2}^{n}\right)\right] \\
& =I\left(\mathcal{S}_{001} ; Y_{2}^{n}\right)+I\left(\mathcal{S}_{010} ; Y_{2}^{n}\right)+\left[I\left(\mathcal{S}_{011} ; X^{n}\right)-I\left(\mathcal{S}_{011} ; Y_{2}^{n}\right)\right] \\
& (b) \\
& \geq I\left(\mathcal{S}_{001} ; Y_{1}^{n}\right)+\left(\mathcal{S}_{010} ; Y_{2}^{n}\right)+\left[I\left(\mathcal{S}_{011} ; X^{n}\right)-I\left(\mathcal{S}_{011} ; Y_{2}^{n}\right)\right] \\
& (c) n \frac{n}{2} \log \frac{1+d_{1}}{D_{001}+d_{1}} \frac{1+d_{2}}{D_{010}+d_{2}} \frac{(1+0)\left(D_{011}+d_{2}\right)}{\left(1+d_{2}\right)\left(D_{011}+0\right)} \\
& \geq \frac{n}{2} \log \frac{D_{4}+D_{2}}{\left(D_{1}+D_{1}\right)\left(D_{2}+D_{2}\right) D_{4}} \\
& \geq \frac{n}{2} \log \frac{D_{2}}{4 D_{1} D_{2} D_{4}} \\
& =n\left(-1+\frac{1}{2} \log D_{1} D_{4}\right)
\end{aligned}
$$

where the subtracted terms in $(a)$ are positive due to the non-negativity of mutual information, $(b)$ is by the data processing inequality, and $(c)$ is due to Lemma 2 and Lemma 3. The same lines of proof can be written for (L5) and (L6).

In order to prove (L9), we have to show two inequalities, one for each term in the maximization expression.

$$
\begin{aligned}
n\left(R_{1}+R_{2}+2 R_{3}+4 \varepsilon\right) \geq & H\left(\mathcal{S}_{001}\right)+H\left(\mathcal{S}_{010}\right)+2 H\left(\mathcal{S}_{100}\right) \\
& (a) \\
\geq & H\left(\mathcal{S}_{001}\right)+H\left(\mathcal{S}_{010}\right)+2 H\left(\mathcal{S}_{100}\right)-H\left(\mathcal{S}_{111} \mid X^{n}\right) \\
& -\left[H\left(\mathcal{S}_{001} \mid Y_{3}^{n}\right)+H\left(\mathcal{S}_{100} \mid Y_{3}^{n}\right)-H\left(\mathcal{S}_{101} \mid Y_{3}^{n}\right)\right] \\
& -\left[H\left(\mathcal{S}_{010} \mid Y_{3}^{n}\right)+H\left(\mathcal{S}_{100} \mid Y_{3}^{n}\right)-H\left(\mathcal{S}_{110} \mid Y_{3}^{n}\right)\right] \\
& -\left[H\left(\mathcal{S}_{101} \mid Y_{6}^{n}\right)+H\left(\mathcal{S}_{110} \mid Y_{6}^{n}\right)-H\left(\mathcal{S}_{111} \mid Y_{6}^{n}\right)\right] \\
= & I\left(\mathcal{S}_{001} ; Y_{3}^{n}\right)+I\left(\mathcal{S}_{010} ; Y_{3}^{n}\right)+2 I\left(\mathcal{S}_{100} ; Y_{3}^{n}\right)+\left[I\left(\mathcal{S}_{101} ; Y_{6}^{n}\right)-I\left(\mathcal{S}_{101} ; Y_{3}^{n}\right)\right] \\
& +\left[I\left(\mathcal{S}_{110} ; Y_{6}^{n}\right)-I\left(\mathcal{S}_{110} ; Y_{3}^{n}\right)\right]+\left[I\left(\mathcal{S}_{111} ; X^{n}\right)-I\left(\mathcal{S}_{111} ; Y_{6}^{n}\right)\right] \\
& (b) \\
\geq & I\left(\mathcal{S}_{001} ; Y_{1}^{n}\right)+I\left(\mathcal{S}_{010} ; Y_{2}^{n}\right)+2 I\left(\mathcal{S}_{100} ; Y_{3}^{n}\right)+\left[I\left(\mathcal{S}_{101} ; Y_{5}^{n}\right)-I\left(\mathcal{S}_{101} ; Y_{3}^{n}\right)\right] \\
& +\left[I\left(\mathcal{S}_{110} ; Y_{6}^{n}\right)-I\left(\mathcal{S}_{110} ; Y_{3}^{n}\right)\right]+\left[I\left(\mathcal{S}_{111} ; X^{n}\right)-I\left(\mathcal{S}_{11} ; Y_{6}^{n}\right)\right] \\
& \geq \frac{n}{2} \log \frac{1+d_{1}}{D_{001}+d_{1}}+\frac{n}{2} \log \frac{1+d_{2}}{D_{010}+d_{2}}+2 \frac{n}{2} \log \frac{1+d_{3}}{D_{100}+d_{3}} \\
& +\frac{n}{2} \log \frac{\left(1+d_{5}\right)\left(D_{101}+d_{3}\right)}{\left(1+d_{3}\right)\left(D_{101}+d_{5}\right)}+\frac{n}{2} \log \frac{\left(1+d_{6}\right)\left(D_{110}+d_{3}\right)}{\left(1+d_{3}\right)\left(D_{110}+d_{6}\right)} \\
& +\frac{n}{2} \log \frac{D_{111}+d_{6}}{\left(1+d_{6}\right) D_{111}} \\
& (d) \\
\geq & \frac{n}{2} \log \frac{1}{D_{1}+D_{1}} \frac{1}{D_{2}+D_{2}} \frac{1}{\left(D_{3}+D_{3}\right)^{2}}+\frac{1}{2} \log \frac{D_{3}}{D_{5}+D_{5}} \frac{D_{3}+D_{6}}{D_{7}} \frac{D_{6}}{D_{7}} \\
= & n\left(-3+\frac{1}{2} \log \frac{1}{D_{1} D_{5} D_{7}}\right) \cdot \\
& \left(-\frac{1}{2}\right)
\end{aligned}
$$

where $(a),(b)$ and $(c)$ are by the same reasons as those in (4), and in $(d)$ all the non-canceled terms in the nominator are replaced by their dominating factor. This proves the first inequality in (L9). The proof of (L7) and (L8) are similar to (6) and based on the same technique.

In order to prove the second inequality in (L9), we start with the same initial step but then subtracting terms differently

$$
\begin{aligned}
n\left(R_{1}+R_{2}+2 R_{3}+4 \varepsilon\right) \geq & H\left(\mathcal{S}_{001}\right)+H\left(\mathcal{S}_{010}\right)+2 H\left(\mathcal{S}_{100}\right) \\
\stackrel{(a)}{\geq} & H\left(\mathcal{S}_{001}\right)+H\left(\mathcal{S}_{010}\right)+2 H\left(\mathcal{S}_{100}\right) \\
& -H\left(\mathcal{S}_{100} \mid Y_{3}^{n}\right)-H\left(\mathcal{S}_{111} \mid X^{n}\right) \\
& -\left[H\left(\mathcal{S}_{001} \mid Y_{2}^{n}\right)+H\left(\mathcal{S}_{010} \mid Y_{2}^{n}\right)-H\left(\mathcal{S}_{011} \mid Y_{2}^{n}\right)\right] \\
& -\left[H\left(\mathcal{S}_{011} \mid Y_{4}^{n}\right)+H\left(\mathcal{S}_{100} \mid Y_{4}^{n}\right)-H\left(\mathcal{S}_{111} \mid Y_{4}^{n}\right)\right]
\end{aligned}
$$


Notice in (5) and (7), we have subtracted different terms, which results in different form of bounds. The particular choice may appears rather arbitrary at the first sight, however, a closer look reveals that for a particular face of the rate region of the A-MLD coding problem, there exists certain independence between the descriptions, when conditioned on some of the source $\left\{V_{1}, V_{2}, \ldots, V_{k}\right\}$. For the asymmetric MD problem, this conditional independence holds approximately with $Y_{k}$ replacing the role of $\left\{V_{1}, V_{2}, \ldots, V_{k}\right\}$.

Continuing the chain of inequalities, we have

$$
\begin{aligned}
n\left(R_{1}+R_{2}+2 R_{3}+4 \varepsilon\right) \geq & I\left(\mathcal{S}_{001} ; Y_{2}^{n}\right)+I\left(\mathcal{S}_{010} ; Y_{2}^{n}\right)+I\left(\mathcal{S}_{100} ; Y_{3}^{n}\right)+I\left(\mathcal{S}_{100} ; Y_{4}^{n}\right) \\
& +\left[I\left(\mathcal{S}_{011} ; Y_{4}^{n}\right)-I\left(\mathcal{S}_{011} ; Y_{2}^{n}\right)\right]+\left[I\left(\mathcal{S}_{111} ; X^{n}\right)-I\left(\mathcal{S}_{111} ; Y_{4}^{n}\right)\right] \\
& (b) \\
\geq & I\left(\mathcal{S}_{001} ; Y_{1}^{n}\right)+I\left(\mathcal{S}_{010} ; Y_{2}^{n}\right)+2 I\left(\mathcal{S}_{100} ; Y_{3}^{n}\right) \\
& +\left[I\left(\mathcal{S}_{011} ; Y_{4}^{n}\right)-I\left(\mathcal{S}_{011} ; Y_{2}^{n}\right)\right]+\left[I\left(\mathcal{S}_{111} ; X^{n}\right)-I\left(\mathcal{S}_{111} ; Y_{4}^{n}\right)\right] \\
& \stackrel{(c)}{\geq} \frac{n}{2} \log \frac{1+d_{1}}{D_{001}+d_{1}}+\frac{n}{2} \log \frac{1+d_{2}}{D_{010}+d_{2}}+2 \frac{n}{2} \log \frac{1+d_{3}}{D_{100}+d_{3}} \\
& +\frac{n}{2} \log \frac{\left(1+d_{4}\right)\left(D_{011}+d_{2}\right)}{\left(1+d_{2}\right)\left(D_{011}+d_{4}\right)}+\frac{n}{2} \log \frac{D_{111}+d_{4}}{\left(1+d_{4}\right) D_{111}} \\
\geq & \frac{n}{2} \log \left(\frac{1}{D_{1}+D_{1}} \frac{1}{D_{2}+D_{2}} \frac{D_{2}}{D_{4}} \frac{1}{\left.D_{3}+D_{3}\right)^{2}}\right) \\
= & n\left(-\frac{5}{D_{4}+D_{4}}+\frac{1}{2} \log \frac{1}{D_{1} D_{3}^{2} D_{7}}\right) .
\end{aligned}
$$

where $(a),(b)$ and $(c)$ are again by the same reasons as in (4). Putting (6) and (8) together, we arrive at (L9).

In the following we will prove two inequalities to bound the sum rate, by showing two different bounds. First, we have

$$
\begin{aligned}
& n\left(R_{1}+R_{2}+R_{3}+3 \varepsilon\right) \geq H\left(\mathcal{S}_{001}\right)+H\left(\mathcal{S}_{010}\right)+H\left(\mathcal{S}_{100}\right) \\
& \text { (a) } \\
& \stackrel{(a)}{\geq}\left(\mathcal{S}_{001}\right)+H\left(\mathcal{S}_{010}\right)+H\left(\mathcal{S}_{100}\right)-H\left(\mathcal{S}_{111} \mid X^{n}\right) \\
& -\frac{1}{2}\left[H\left(\mathcal{S}_{001} \mid Y_{2}^{n}\right)+H\left(\mathcal{S}_{010} \mid Y_{2}^{n}\right)-H\left(\mathcal{S}_{011} \mid Y_{2}^{n}\right)\right] \\
& -\frac{1}{2}\left[H\left(\mathcal{S}_{001} \mid Y_{3}^{n}\right)+H\left(\mathcal{S}_{100} \mid Y_{3}^{n}\right)-H\left(\mathcal{S}_{101} \mid Y_{3}^{n}\right)\right] \\
& -\frac{1}{2}\left[H\left(\mathcal{S}_{010} \mid Y_{3}^{n}\right)+H\left(\mathcal{S}_{100} \mid Y_{3}^{n}\right)-H\left(\mathcal{S}_{110} \mid Y_{3}^{n}\right)\right] \\
& -\frac{1}{2}\left[H\left(\mathcal{S}_{011} \mid Y_{4}^{n}\right)+I\left(\mathcal{S}_{101} \mid Y_{4}^{n}\right)+I\left(\mathcal{S}_{110} \mid Y_{4}^{n}\right)-2 H\left(\mathcal{S}_{111} \mid Y_{4}^{n}\right)\right] \\
& =\frac{1}{2}\left[I\left(\mathcal{S}_{001} ; Y_{2}^{n}\right)+I\left(\mathcal{S}_{001} ; Y_{3}^{n}\right)+I\left(\mathcal{S}_{010} ; Y_{2}^{n}\right)+I\left(\mathcal{S}_{010} ; Y_{3}^{n}\right)+2 I\left(\mathcal{S}_{100} ; Y_{3}^{n}\right)\right] \\
& +\frac{1}{2}\left[I\left(\mathcal{S}_{011} ; Y_{4}^{n}\right)-I\left(\mathcal{S}_{011} ; Y_{2}^{n}\right)\right]+\frac{1}{2}\left[I\left(\mathcal{S}_{101} ; Y_{4}^{n}\right)-I\left(\mathcal{S}_{101} ; Y_{3}^{n}\right)\right] \\
& +\frac{1}{2}\left[I\left(\mathcal{S}_{110} ; Y_{4}^{n}\right)-I\left(\mathcal{S}_{110} ; Y_{3}^{n}\right)\right]+\left[I\left(\mathcal{S}_{111} ; X^{n}\right)-I\left(\mathcal{S}_{111} ; Y_{4}^{n}\right)\right] \\
& \text { (b) } \\
& \stackrel{(b)}{\geq}\left(\mathcal{S}_{001} ; Y_{1}^{n}\right)+I\left(\mathcal{S}_{010} ; Y_{2}^{n}\right)+I\left(\mathcal{S}_{100} ; Y_{3}^{n}\right) \\
& +\frac{1}{2}\left[I\left(\mathcal{S}_{011} ; Y_{4}^{n}\right)-I\left(\mathcal{S}_{011} ; Y_{2}^{n}\right)\right]+\frac{1}{2}\left[I\left(\mathcal{S}_{101} ; Y_{4}^{n}\right)-I\left(\mathcal{S}_{101} ; Y_{3}^{n}\right)\right] \\
& +\frac{1}{2}\left[I\left(\mathcal{S}_{110} ; Y_{4}^{n}\right)-I\left(\mathcal{S}_{110} ; Y_{3}^{n}\right)\right]+\left[I\left(\mathcal{S}_{111} ; X^{n}\right)-I\left(\mathcal{S}_{111} ; Y_{4}^{n}\right)\right] \\
& \stackrel{\text { (c) }}{\geq} \frac{n}{2} \log \left(\frac{1+d_{1}}{D_{001}+d_{1}} \frac{1+d_{2}}{D_{010}+d_{2}} \frac{1+d_{3}}{D_{100}+d_{3}}\right) \\
& +\frac{n}{4} \log \frac{\left(1+d_{4}\right)\left(D_{011}+d_{2}\right)}{\left(1+d_{2}\right)\left(D_{011}+d_{4}\right)}+\frac{n}{4} \log \frac{\left(1+d_{4}\right)\left(D_{101}+d_{3}\right)}{\left(1+d_{3}\right)\left(D_{101}+d_{4}\right)} \\
& +\frac{n}{4} \log \frac{\left(1+d_{4}\right)\left(D_{110}+d_{3}\right)}{\left(1+d_{3}\right)\left(D_{110}+d_{4}\right)}+\frac{n}{2} \log \frac{D_{111}+d_{4}}{\left(1+d_{4}\right) D_{111}} \\
& \geq \frac{n}{4} \log \frac{1}{\left(D_{1}+D_{1}\right)^{2}\left(D_{2}+D_{2}\right)^{2}\left(D_{3}+D_{3}\right)^{2}} \frac{D_{2}}{D_{4}+D_{4}} \frac{D_{3}}{D_{4}+D_{4}}+\frac{n}{4} \log \frac{D_{3}}{D_{4}+D_{4}} \frac{D_{4}^{2}}{D_{7}^{2}} \\
& =n\left(-\frac{9}{4}+\frac{1}{4} \log \frac{1}{D_{1}^{2} D_{2} D_{4} D_{7}^{2}}\right)
\end{aligned}
$$


where all the subtracted terms in $(a)$ are positive by conditional Han's inequality; $(b)$ is due to the data processing inequality; and $(c)$ follows from Lemma 2 and Lemma 3. This proves the inequality corresponding to the first term in the maximization expression.

On the other hand, by rearranging the terms, we can write

$$
\begin{aligned}
n\left(R_{1}+R_{2}+R_{3}+3 \varepsilon\right) \geq & H\left(\mathcal{S}_{001}\right)+H\left(\mathcal{S}_{010}\right)+H\left(\mathcal{S}_{100}\right) \\
& \stackrel{(a)}{\geq} H\left(\mathcal{S}_{001}\right)+H\left(\mathcal{S}_{010}\right)+H\left(\mathcal{S}_{100}\right)-H\left(\mathcal{S}_{111} \mid X^{n}\right) \\
& -\left[H\left(\mathcal{S}_{001} \mid Y_{2}^{n}\right)+H\left(\mathcal{S}_{010} \mid Y_{2}^{n}\right)-H\left(\mathcal{S}_{011} \mid Y_{2}^{n}\right)\right] \\
& -\left[H\left(\mathcal{S}_{100} \mid Y_{4}^{n}\right)+H\left(\mathcal{S}_{011} \mid Y_{4}^{n}\right)-H\left(\mathcal{S}_{111} \mid Y_{4}^{n}\right)\right] \\
= & I\left(\mathcal{S}_{001} ; Y_{2}^{n}\right)+I\left(\mathcal{S}_{010} ; Y_{2}^{n}\right)+I\left(\mathcal{S}_{100} ; Y_{4}^{n}\right) \\
& +\left[I\left(\mathcal{S}_{011} ; Y_{4}^{n}\right)-I\left(\mathcal{S}_{011} ; Y_{2}^{n}\right)\right]+\left[I\left(\mathcal{S}_{111} ; X^{n}\right)-I\left(\mathcal{S}_{111} ; Y_{4}^{n}\right)\right] \\
& (b) \\
\geq & I\left(\mathcal{S}_{001} ; Y_{1}^{n}\right)+I\left(\mathcal{S}_{010} ; Y_{2}^{n}\right)+I\left(\mathcal{S}_{100} ; Y_{3}^{n}\right) \\
& +\left[I\left(\mathcal{S}_{011} ; Y_{4}^{n}\right)-I\left(\mathcal{S}_{011} ; Y_{2}^{n}\right)\right]+\left[I\left(\mathcal{S}_{111} ; X^{n}\right)-I\left(\mathcal{S}_{111} ; Y_{4}^{n}\right)\right. \\
& (c) \\
\geq & \frac{n}{2} \log \left(\frac{1+d_{1}}{D_{001}+d_{1}} \frac{1+d_{2}}{D_{010}+d_{2}} \frac{1+d_{3}}{D_{100}+d_{3}}\right) \\
& +\frac{n}{2} \log \frac{\left(1+d_{4}\right)\left(D_{011}+d_{2}\right)}{\left(1+d_{2}\right)\left(D_{011}+d_{4}\right)}+\frac{n}{2} \log \frac{D_{111}+d_{4}}{\left(1+d_{4}\right) D_{111}} \\
\geq & \frac{n}{2} \log \left(\frac{1}{D_{1}+D_{1}} \frac{1}{D_{2}+D_{2}} \frac{1}{D_{3}+D_{3}} \frac{D_{2}}{D_{4}+D_{4}} \frac{D_{4}}{D_{7}}\right) \\
= & n\left(-2+\frac{1}{2} \log \frac{1}{D_{1} D_{3} D_{7}}\right) \cdot
\end{aligned}
$$

where again positivity of the subtracted terms in $(a)$ follows from the conditional Han's inequality; data processing inequality implies $(b)$; and $(c)$ is due to Lemma 2 and Lemma 3. Finally, (9) and (10) together imply (L10).

\section{An APPRoximate Characterization of $\mathcal{R}(\mathbf{D})$}

Summarizing the results of Theorem 2 and Theorem 3 gives

$$
\overline{\mathcal{R}}(\mathbf{D}) \subseteq \mathcal{R}(\mathbf{D}) \subseteq \underline{\mathcal{R}}(\mathbf{D}) .
$$

Therefore, we have bounded the boundary of the admissible rate region between two sets of hyperplanes, which are pairwise parallel. For each pair of parallel planes, we can compute the distance between them pair-wise. Denote by $\delta_{(x, y, z)}$ the Euclidean distance between two parallel planes which are orthogonal to the vector $(x, y, z)$. Then it follows

$$
\begin{aligned}
& \delta_{(1,0,0)}=0 \\
& \delta_{(1,1,0)} \leq \frac{1}{\sqrt{2}}=0.7071 \\
& \delta_{(2,1,1)} \leq \frac{3}{\sqrt{6}}=1.2247 \\
& \delta_{(1,1,1)} \leq \frac{9}{4 \sqrt{3}}=1.2990
\end{aligned}
$$

where the denominator are the normalizing factor. This shows that the inner and outer bounds provide a characterization of the admissible rate region within at most 1.3 bits in the worst case. Fig. 4 shows the inner and outer bounds for the case $D_{2} D_{4} \geq D_{3}^{2} \geq D_{2} D_{5}$.

\section{Conclusion}

We examined the asymmetric three description problem for the Gaussian source under the MSE distortion measure. Inner and outer bounds for the achievable rate region are given, and the difference between them are shown to be small. Though the general asymmetric Gaussian MD rate distortion region is hard to characterize, it is rather satisfying to see that a simple coding architecture is almost optimal. The A-MLD coding problem plays a key role in establishing these results, which further strengthens the connection between MLD coding and MD problem. 


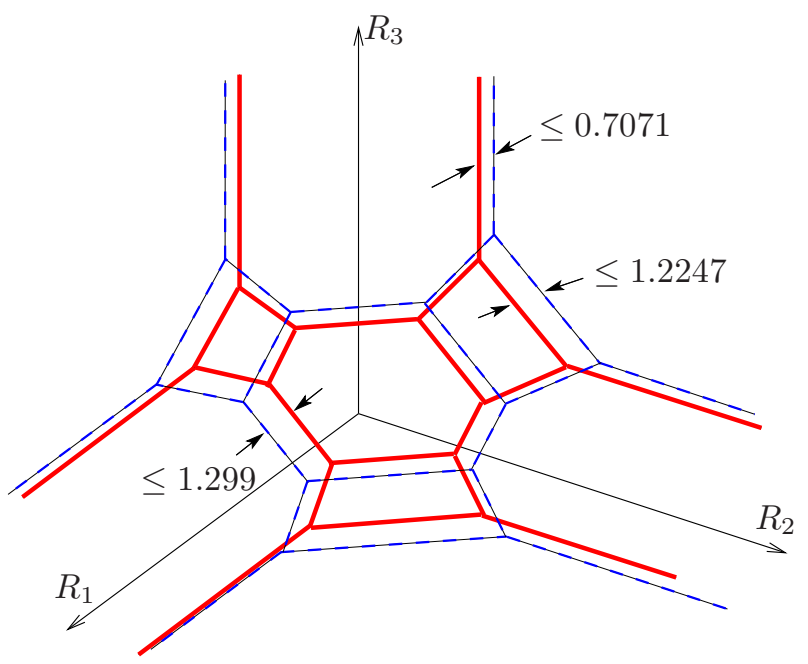

Fig. 4. The inner and outer bound for the case $D_{2} D_{4} \geq D_{3}^{2} \geq D_{2} D_{5}$.

\section{REFERENCES}

[1] A. El Gamal and T. Cover, "Achievable Rates for Multiple Descriptions," IEEE Transaction on Information Theory, Vol. 28, no. 6, pp. 851-857, Nov. 1982.

[2] L. Ozarow, "On a Source-Coding Problem with Two Channels and Three Receivers," Bell System Technical Journal, vol. 59, no. 10, pp. 1909-1921, Dec. 1980.

[3] Z. Zhang and T. Berger, "New Results in Binary Multiple Descriptions," IEEE Transaction on Information Theory, Vol. 33, no. 4, pp. 502-821, July 1987.

[4] S. Mohajer, C. Tian, and S. N. Diggavi, "Asymmetric multi-level diversity coding," to appear, Data Compression Conference, Snowbird Utah, Mar. 2007.

[5] C. Tian, S. Mohajer, and S. N. Diggavi, "On the symmetric Gaussian multiple descriptions rate-distortion function," to appear, Data Compression Conference, Snowbird Utah, Mar. 2007.

[6] J. R. Roche, R. W. Yeung, and K. P. Hau, "Symmetrical multilevel diversity coding," IEEE Trans. Information Theory, vol. 43, no. 5, pp. 1059-1064, May 1997.

[7] R. W. Yeung and Z. Zhang, "On symmetrical multilevel diversity coding," IEEE Trans. Information Theory, vol. 45, no. 2, pp. 609-621, Mar. 1999.

[8] T. M. Cover and J. A. Thomas, Elements of information theory, New York: Wiley, 1991.

[9] C. Tian, S. Mohajer, and S. N. Diggavi, "Approximating the Gaussian multiple description rate region under symmetric distortion constraints," EPFL technical report, available from http://licos.epfl.ch, 2008.; submitted to ISIT 2008.

[10] W. H. R. Equitz and T. M. Cover, "Successive refinement of information," IEEE Trans. Information Theory, vol. 37, no. 2, pp. 269-275, Mar. 1991.

[11] R. Puri, S.S. Pradhan, and K. Ramchandran, " $n$-channel symmetric multiple descriptions - Part II: an achievable rate-distortion region," IEEE Trans. Information Theory, vol. 51, pp. 1377-1392, Apr. 2005.

[12] S. Diggavi and T. M. Cover, "Worst additive noise under covariance constraints," IEEE Trans. Information Theory, vol. 47, no. 7, pp. 3072-3081, Nov. 2001. 